\title{
Language development of three to twelve-year-old twins compared to singletons
}

Evelien D'haeseleer, Eline Geenens, Sarah Parmentier, Kristiane Van Lierde

Evelien D'haeseleer

Ghent University, Royal Conservatory Brussels, Belgium
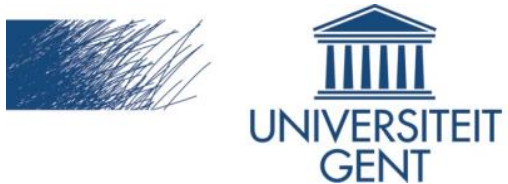

Speech, Language and Hearing Sciences UNIVERSITEIT GENT

\section{No disclosures}

Evelien D'haeseleer, Eline Geenens, Sarah Parmentier, Kristiane Van Lierde 


\section{$\widehat{\text { IIIIII }}$ \\ UNIVERSITEIT \\ GENT}

\section{Introduction}

\section{- Language development in twins tend to lag behind}

\begin{tabular}{|l|l|l|l|}
\hline Authors & subjects & Language aspects & Results \\
\hline Day, 1932 & $\begin{array}{l}80 \text { twins } \\
\text { Age range: 2-5 y }\end{array}$ & $\begin{array}{l}\text { Expressive vocabulary and syntax } \\
\text { Production of the first words }\end{array}$ & $\begin{array}{l}\text { Twins scored weak for expressive } \\
\text { vocabulary and syntax. Twins } \\
\text { produced their first words at a } \\
\text { mean age of 25 months. }\end{array}$ \\
\hline Davis, 1937 & $\begin{array}{l}436 \text { twins and } \\
\text { singletons } \\
\text { Age range: 5;6y- } \\
9 ; 6 y .\end{array}$ & $\begin{array}{l}\text { Articulation, expressive vocabulary } \\
\text { and syntax. }\end{array}$ & $\begin{array}{l}\text { Twins scored lower for } \\
\text { articulation and expressive } \\
\text { vocabulary. No differences } \\
\text { between twins and singletons for } \\
\text { syntax. }\end{array}$ \\
\hline
\end{tabular}

\begin{tabular}{|l|l|l|l|}
\hline Authors & subjects & Language aspects & Results \\
\hline Day, 1932 & $\begin{array}{l}80 \text { twins } \\
\text { Age range: 2-5 y }\end{array}$ & $\begin{array}{l}\text { Expressive vocabulary and syntax } \\
\text { Production of the first words }\end{array}$ & $\begin{array}{l}\text { Twins scored weak for expressive } \\
\text { vocabulary and syntax. Twins } \\
\text { produced their first words at a } \\
\text { mean age of 25 months. }\end{array}$ \\
\hline Davis, 1937 & $\begin{array}{l}436 \text { twins and } \\
\text { singletons } \\
\text { Age range: 5;6y- } \\
9 ; 6 y .\end{array}$ & $\begin{array}{l}\text { Articulation, expressive vocabulary } \\
\text { and syntax. }\end{array}$ & $\begin{array}{l}\text { Twins scored lower for } \\
\text { articulation and expressive } \\
\text { vocabulary. No differences } \\
\text { between twins and singletons for } \\
\text { syntax. }\end{array}$ \\
\hline
\end{tabular}




\begin{tabular}{|c|c|c|c|}
\hline & $\begin{array}{c}\text { UNIVERSITEIT } \\
\text { GENT }\end{array}$ & \multicolumn{2}{|c|}{ Speech, Language and Hearing Sciences } \\
\hline Authors & subjects & Language aspects & Results \\
\hline $\begin{array}{l}\text { Rutter et al., } \\
2003\end{array}$ & $\begin{array}{l}96 \text { twins, } 98 \\
\text { singletons } \\
\text { Age: at } 20 \text { months and } \\
3 y\end{array}$ & $\begin{array}{l}\text { Receptive and Expressive language } \\
\text { skills using The McArthur } \\
\text { Communicative Development } \\
\text { Inventory (at } 20 \text { months) and the Pre- } \\
\text { School Language Scales and } \\
\text { McCarthy Scales of Children's } \\
\text { Abilities (at } 36 \text { months) }\end{array}$ & $\begin{array}{l}\text { Language delay in twins of } 1.7 \\
\text { months at the age of } 20 \text { months } 3.1 \\
\text { months at the age of } 3 \text { years. }\end{array}$ \\
\hline $\begin{array}{l}\text { Gucuyener et } \\
\text { al., } 2011\end{array}$ & $\begin{array}{l}162 \text { twins, } 124 \\
\text { singletons } \\
\text { Age: } 5 y\end{array}$ & $\begin{array}{l}\text { Expressive vocabulary using the } \\
\text { Peabody Picture Vocabulary Test }\end{array}$ & $\begin{array}{l}\text { Even after excluding premature } \\
\text { twins, twins performed worse than } \\
\text { singletons and twin girls performed } \\
\text { better than twin boys. Preterm twins } \\
\text { performed worse than term twins. }\end{array}$ \\
\hline Rice et al., 2013 & $\begin{array}{l}473 \text { twins pairs } \\
\text { Age: } 24 \text { months }\end{array}$ & $\begin{array}{l}\text { Vocabulary and grammar using the } \\
\text { Mac Arthur Communicative } \\
\text { Development Inventories: words and } \\
\text { sentences }\end{array}$ & $\begin{array}{l}\text { Twins had lower average scores } \\
\text { than norms for singletons. } \\
\text { Monozygotic twins and boys had } \\
\text { lower scores than dizygotic twins } \\
\text { and girls. }\end{array}$ \\
\hline
\end{tabular}

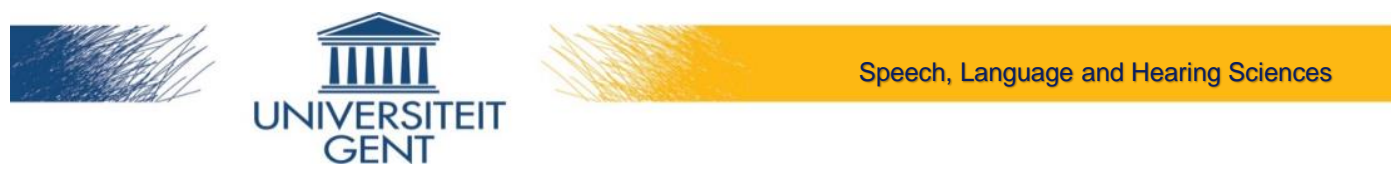

\section{Shortcomings in the literature}

- Mostly based on older studies

- Especially young children

- Parental questionnaires

- Psychological, cognitive test batteries

$\rightarrow$ Not appropriate

$\rightarrow$ Standardized language batteries are more suitable 


\section{$\widehat{\underline{\text { IIIIII }}}$ \\ UNIVERSITEIT}

Speech, Language and Hearing Sciences

\section{Purpose}

To compare expressive and receptive language skills of three to twelve-year-old twins with matched singletons using a standardized language battery, the CELF-4-NL

To investigate the language differences between singletons and twins with a normal gestational age at birth by excluding preterm born children.

To investigate whether the language differences between twins and singletons change with increasing age.

Evelien D'haeseleer

Ghent University, Royal Conservatory Brussels, Belgium

\section{UNIVERSITEIT
GENT}

\section{Methods - subjects}

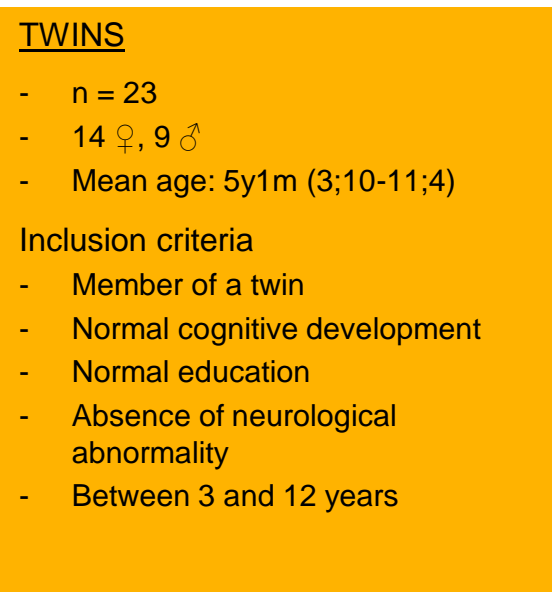

\begin{tabular}{ll}
\multicolumn{2}{l}{ SINGLETONS } \\
- $\mathrm{n}=23$ \\
- $\quad 14$, 90 \\
- & Mean age: $5 y 1 \mathrm{~m}(3 ; 9-11 ; 4)$ \\
Inclusion criteria \\
- $\quad$ Singletons matched for age (+- 3 \\
- $\quad$ Nonths) and gender \\
- $\quad$ Normal education \\
- $\quad$ Absence of neurological \\
$\quad$ abnormality \\
- Between 3 and 12 years
\end{tabular}

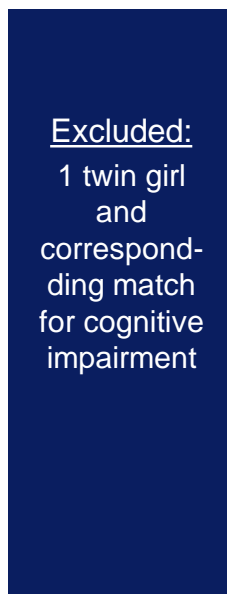

Evelien D'haeseleer

Ghent University, Royal Conservatory Brussels, Belgium 


\section{$\widehat{\text { IIIIII }}$ \\ UNIVERSITEIT \\ GENT}

\section{Methods - language assessment}

- Clinical Evaluation of Language Fundamentals (CELF-A-NL)

- For children $>6$ years

- Core Language Index (CLI)

- Receptive Language Index (RLI)

- Expressive Language Index (ELI)

\section{- CELF Preschool-II-NL}

- For children $\leq 6$ years

- Core Language Index (CLI)

- Parental questionnaire

- Neonatal and socio demographic data

- Receptive Language Index (RLI)

- Expressive Language Index (ELI)

Evelien D'haeseleer

Ghent University, Royal Conservatory Brussels, Belgium

\section{UNIVERSITEIT}

GENT

\section{Methods - statistical analysis}

- Data $\rightarrow$ percentile ranks

- Comparison data twins and singletons

- Wilcoxon matched-pairs signed-ranks test

- Comparison data term twins and singletons

- Exclusion of all preterm born children

- Wilcoxon matched-pairs signed-ranks test

- Correlation between age and language difference between each pair of twins and singletons

- Spearman correlation 


\section{$\widehat{\text { IIIIII }}$ \\ UNIVERSITEIT \\ GENT}

\section{Results}

\section{Neonatal and socio demographic data}

\section{- Socio-economic status}

- Maternal education level

- Chi square test: $p=0.064$

\begin{tabular}{|l|l|l|l|}
\hline Education level & $\begin{array}{l}\text { Twins } \\
n(\%)\end{array}$ & $\begin{array}{l}\text { Singletons } \\
n(\%)\end{array}$ & $\begin{array}{l}\text { Total group } \\
n(\%)\end{array}$ \\
\hline Secondary school & $7(15.2 \%)$ & $1(2.2 \%)$ & $8(17.4 \%)$ \\
\hline $\begin{array}{l}\text { Tertiary education } \\
\text { (College) }\end{array}$ & $10(21.7 \%)$ & $13(28.2 \%)$ & $23(50 \%)$ \\
\hline $\begin{array}{l}\text { Tertiary education } \\
\text { (University) }\end{array}$ & $6(13.0 \%)$ & $9(19.6 \%)$ & $15(32.6 \%)$ \\
\hline
\end{tabular}

Evelien D'haeseleer

Ghent University, Royal Conservatory Brussels, Belgium

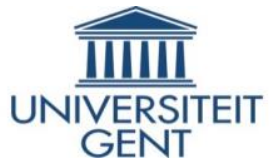

\section{Results}

- Neonatal characteristics

\begin{tabular}{|c|c|c|c|c|c|c|c|c|c|}
\hline & \multicolumn{4}{|c|}{ Twins } & \multicolumn{4}{|c|}{ Singletons } & \multirow[b]{2}{*}{ p-value } \\
\hline & Mean & $\mathrm{SD}$ & Min. & Max. & Mean & SD & Min. & Max. & \\
\hline $\begin{array}{l}\text { Mean gestational age } \\
\text { at birth (weeks) }\end{array}$ & 35.0 & 3.6 & 27.0 & 38.0 & 39.4 & 1.1 & 37.0 & 41.0 & $<0.001$ \\
\hline Birth weight (g) & 2519.4 & 472.9 & 1700.0 & 3400 & 3540.0 & 375.2 & 2860 & 4260.0 & $<0.001$ \\
\hline Birth lenght $(\mathrm{cm})$ & 47.5 & 3.0 & 40.0 & 52 & 50.6 & 2.0 & 46.0 & 57.0 & $<0.001$ \\
\hline $\begin{array}{l}\text { Age mother at birth } \\
\text { (y) }\end{array}$ & 31.0 & 3.0 & 27.0 & 36 & 29.8 & 4.0 & 24.0 & 40.0 & 0.097 \\
\hline
\end{tabular}

Evelien D'haeseleer

Ghent University, Royal Conservatory Brussels, Belgium 


\section{$\widehat{\text { IIIIII }}$ \\ UNIVERSITEIT \\ GENT}

\section{Results}

- Comparison twins and singletons

\begin{tabular}{|c|c|c|c|c|c|c|c|c|c|c|c|}
\hline \multirow{2}{*}{$\begin{array}{l}\text { Language } \\
\text { indexes }\end{array}$} & \multicolumn{5}{|c|}{ Twins } & \multicolumn{5}{|c|}{ Singletons } & \multirow[b]{2}{*}{$p$-value } \\
\hline & $n$ & Mean & SD & $\begin{array}{c}\mathrm{Mi} \\
\mathrm{n}\end{array}$ & Max & $n$ & Mean & SD & Min & Max & \\
\hline CLI & 23 & 38.11 & 27.35 & 4.8 & 88.5 & 23 & 57.21 & 16.44 & 9.1 & 78.8 & 0.009 \\
\hline RLI & 23 & 44.21 & 29.15 & 5.5 & 95.8 & 23 & 68.00 & 25.10 & 4.2 & 95.8 & 0.011 \\
\hline ELI & 23 & 33.02 & 24.56 & 1.0 & 74.8 & 23 & 52.36 & 17.82 & 6.3 & 80.7 & 0.009 \\
\hline
\end{tabular}

Evelien D'haeseleer

Ghent University, Royal Conservatory Brussels, Belgium

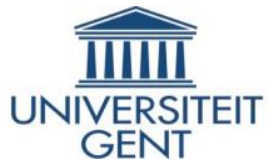

\section{Results}

- Comparison term twins - singletons

\begin{tabular}{|l|c|c|c|c|c|c|c|c|c|c|c|}
\hline $\begin{array}{l}\text { language } \\
\text { indexes }\end{array}$ & \multicolumn{5}{|c|}{ Twins } & \multicolumn{5}{c|}{ Singletons } & \\
\cline { 3 - 14 } & $\mathrm{n}$ & Mean & SD & Min & Max & $\mathrm{n}$ & Mean & SD & Min & Max & $\begin{array}{c}\mathrm{p} \text { - } \\
\text { value }\end{array}$ \\
\hline CLI & 10 & 38.00 & 27.45 & 4.8 & 88.5 & 10 & 48.51 & 17.32 & 9.1 & 70.3 & 0.445 \\
\hline RLI & 10 & 40.82 & 32.01 & 5.5 & 88.5 & 10 & 59.82 & 31.86 & 4.2 & 95.8 & 0.203 \\
\hline ELI & 10 & 31.22 & 19.73 & 6.3 & 60.5 & 10 & 49.04 & 23.00 & 6.3 & 80.7 & 0.047 \\
\hline
\end{tabular}

Evelien D'haeseleer

Ghent University, Royal Conservatory Brussels, Belgium 


\section{$\widehat{\text { IIIIII }}$ \\ UNIVERSITEIT \\ GENT}

Speech, Language and Hearing Sciences

\section{Results}

\section{- Correlation age - language difference singletons/twins}

\begin{tabular}{|l|l|l|}
\hline language indexes & $\begin{array}{l}\text { Correlation } \\
\text { coefficient }\end{array}$ & p-value \\
\hline CLI & 0.290 & 0.179 \\
\hline RLI & 0.259 & 0.233 \\
\hline ELI & 0.122 & 0.580 \\
\hline
\end{tabular}

Evelien D'haeseleer

Ghent University, Royal Conservatory Brussels, Belgium

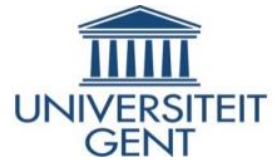

Speech, Language and Hearing Sciences

\section{Discussion}

- Twins score sign. lower than singletons for expressive and receptive language skills

- Mean difference: pc 19,1

- Mean scores correspond with normal language skills in both groups

- Language delay is mild!

- Lower for all language aspects: semantics, morphology, syntax

- No information about pragmatic skills!

- // Rutter et al. (2003), Gucuyener et al., 2011; Rice et al. (2014) 


\section{$\widehat{\text { IIIIII }}$ \\ UNIVERSITEIT \\ GENT}

\section{Discussion}

\section{- Possible causes?}

- No socio-economic differences

- No differences in maternal education level between both groups

\section{- Preterm birth}

- Language delay even between term twins and singletons

- Difference is not significant for the CLI and the RLI

- Small sample size

$\rightarrow$ Preterm birth cannot be (the only) cause of language delay in twins

\section{$\widehat{\text { IIIIII }}$ \\ UNIVERSITEIT \\ GENT}

\section{Discussion}

- Possible causes?

- Differences in postnatal experiences?

- Different patterns in family interaction

- Parents need to divide their attention

- Less one-to-one interactions

- Additional stresses (Rutter et al., 2003)

- Parentese

- Less and less complex (Conway et al., 1980)

- More directed at both twins

- Language between siblings

- more interactions with the same developmental level (Rutter et al., 2003) 


\section{$\widehat{\text { IIIIII }}$ \\ UNIVERSITEIT \\ GENT}

- Cryptophasia ('private language'):

- Phenomenon of an autonomous language developed by twins that only the children understand.

- Developmental phenomenon occurring in both twins and singletons

- Prevalence is higher in twins (Thorpe et al. 2001)

- More an early indication than a cause

Evelien D'haeseleer

Ghent University, Royal Conservatory Brussels, Belgium

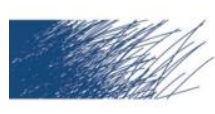

\section{$\widehat{\text { IIIIII }}$ \\ UNIVERSITEIT \\ GENT}

\section{Discussion}

\section{- Temporary phenomenon?}

- Literature: not clear, rather a temporary delay,

- No correlation between age and the difference in language skills between twins and singletons

- Language delay does not seem to decrease over time

- Further research in older twins and singletons!

Recent study

Evelien D'haeseleer

Ghent University, Royal Conservatory Brussels, Belgium 


\section{$\widehat{\text { IIIIII }}$ \\ UNIVERSITEIT \\ GENT}

\section{Limitations}

- Small sample size

- Absence of IQ data

- Number of siblings should be taken into account

- Pragmatic skills and spontaneous language should be further investigated.

\section{$\widehat{\text { IIIIII }}$ \\ UNIVERSITEIT}

\section{Reference}

D'haeseleer, E., Geenens, E., Parmentier, S., Corthals, P., Van Lierde, K. (2014). Language development of 3 to 12 year old twins compared to singletons. Folia Phoniatrica et Logopaedica, in press. 